\title{
Parallel Solution of Cardiac Reaction-Diffusion Models
}

\author{
Luca F. Pavarino ${ }^{1}$ and Piero Colli Franzone ${ }^{2}$ \\ 1 Università di Milano, Dept. of Mathematics (www.mat.unimi.it/〜pavarino) \\ 2 Università di Pavia, Dept. of Mathematics (www-dimat.unipv.it)
}

\begin{abstract}
Summary. We present and study a parallel iterative solver for reaction-diffusion systems in three dimensions arising in computational electrocardiology, such as the Bidomain and Monodomain models. The models include intramural fiber rotation and anisotropic conductivity coefficients that can be fully orthotropic or axially symmetric around the fiber direction. These cardiac models are coupled with a membrane model for the ionic currents, consisting of a system of ordinary differential equations. The solver employs structured isoparametric $Q_{1}$ finite elements in space and a semi-implicit adaptive method in time. Parallelization and portability are based on the PETSc parallel library and large-scale computations with up to $O\left(10^{7}\right)$ unknowns have been run on parallel computers. These simulation of the full Bidomain model (without operator or variable splitting) for a full cardiac cycle are, to our knowledge, among the most complete in the available literature.
\end{abstract}

\section{The cardiac Bidomain and Monodomain models}

Cardiac tissue is traditionally modeled as an arrangement of cardiac fibers that rotate counterclockwise from the epicardium to the endocardium, (see Streeter [1979]). Moreover, from LeGrice and et al. [1995], the cardiac tissue has a laminar organization that can be modeled as a set of muscle sheets running radially from epi to endocardium. Therefore, at any point $\mathbf{x}$, it is possible to identify a triplet of orthonormal principal axes $\mathbf{a}_{l}(\mathbf{x}), \mathbf{a}_{t}(\mathbf{x}), \mathbf{a}_{n}(\mathbf{x})$, with $\mathbf{a}_{l}(\mathbf{x})$ parallel to the local fiber direction, $\mathbf{a}_{t}(\mathbf{x})$ and $\mathbf{a}_{n}(\mathbf{x})$ tangent and orthogonal to the radial laminae respectively and both being transversal to the fiber axis. The macroscopic Bidomain model represents the cardiac tissue as the superposition of two anisotropic continuous media, the intra (i) and extra (e) cellular media, coexisting at every point of the tissue and connected by a distributed continuous cellular membrane; see Keener and Sneyd [1998]. The anisotropic conductivity properties of the tissue are described by the conductivity coefficients in the intra and extracellular media $\sigma_{l}^{i, e}, \sigma_{t}^{i, e}, \sigma_{n}^{i, e}$, measured along the corresponding directions $\mathbf{a}_{l}, \mathbf{a}_{t}, \mathbf{a}_{n}$, and by the conductivity tensors $D_{i}(\mathbf{x})$ and $D_{e}(\mathbf{x})$ 


$$
D_{i, e}=\sigma_{l}^{i, e} \mathbf{a}_{l} \mathbf{a}_{l}^{T}+\sigma_{t}^{i, e} \mathbf{a}_{t} \mathbf{a}_{t}^{T}+\sigma_{n}^{i, e} \mathbf{a}_{n} \mathbf{a}_{n}^{T} .
$$

When the media are axially isotropic, i.e. when $\sigma_{n}^{i, e}=\sigma_{t}^{i, e}$, we have $D_{i, e}=$ $\sigma_{t}{ }^{i, e} I+\left(\sigma_{l}{ }^{i, e}-\sigma_{t}{ }^{i, e}\right) \mathbf{a}_{l} \mathbf{a}_{l}^{T}$. The intra and extracellular electric potentials $u_{i}, u_{e}$ in an insulated cardiac domain $H$ are described in the Bidomain model by a reaction-diffusion system coupled with a system of ODEs for the ionic gating variables $w$. Given the applied currents per unit volume $I_{a p p}^{i, e}$, satisfying the compatibility condition $\int_{H} I_{a p p}^{i} \mathrm{dx}=\int_{H} I_{a p p}^{e} \mathrm{dx}$, the initial conditions $v_{0}, w_{0}$, then $u_{i}, u_{e}, w$ satisfy the system:

$$
\left\{\begin{array}{l}
c_{m} \frac{\partial v}{\partial t}-\operatorname{div}\left(D_{i} \nabla u_{i}\right)+I_{i o n}(v, w)=I_{a p p}^{i} \\
-c_{m} \frac{\partial v}{\partial t}-\operatorname{div}\left(D_{e} \nabla u_{e}\right)-I_{i o n}(v, w)=-I_{a p p}^{e} \\
\frac{\partial w}{\partial t}-R(v, w)=0, \quad v(t)=u_{i}(t)-u_{e}(t) \\
\mathbf{n}^{T} D_{i} \nabla u_{i}=0, \quad \mathbf{n}^{T} D_{e} \nabla u_{e}=0 \\
v(\mathbf{x}, 0)=v_{0}(\mathbf{x}), \quad w(\mathbf{x}, 0)=w_{0}(\mathbf{x})
\end{array}\right.
$$

where $c_{m}=\chi * C_{m}, I_{i o n}=\chi * i_{i o n}$, with $\chi$ the ratio of membrane surface area per tissue volume, $C_{m}$ the membrane capacitance and $i_{i o n}$ the ionic current of the membrane per unit area. Existence and regularity results for this degenerate system can be found in Colli Franzone and Savaré [2002]. The system uniquely determines $v$, while the potentials $u_{i}$ and $u_{e}$ are defined only up to a same additive time-dependent constant related to the reference potential, chosen to be the average extracellular potential in the cardiac volume by imposing $\int_{H} u_{e} \mathrm{dx}=0$.

If the two media have equal anisotropy ratio, i.e. $D_{i}=\lambda D_{e}$ with $\lambda$ constant, then the Bidomain system reduces to the Monodomain model consisting in a parabolic reaction-diffusion equation for the transmembrane potential $v$ coupled with a system of ODEs for the gating variables:

$$
\left\{\begin{array}{l}
c_{m} \frac{\partial v}{\partial t}-\operatorname{div}\left(D_{m}(\mathbf{x}) \nabla v\right)+I_{i o n}(v, w)=I_{a p p}^{m}, \\
\frac{\partial w}{\partial t}-R(v, w)=0, \quad w(\mathbf{x}, 0)=w_{0}(\mathbf{x}), \\
\mathbf{n}^{T} D_{m} \nabla v=0, \quad v(\mathbf{x}, 0)=v_{0}(\mathbf{x}),
\end{array}\right.
$$

where $D_{m}=\sigma_{l} \mathbf{a}_{l} \mathbf{a}_{l}^{T}+\sigma_{t} \mathbf{a}_{t} \mathbf{a}_{t}^{T}+\sigma_{n} \mathbf{a}_{n} \mathbf{a}_{n}^{T}$, with $\sigma_{l, t, n}=\lambda \sigma_{l, t, n}^{i} /(1+\lambda)$ and $I_{a p p}^{m}=\left(\lambda I_{a p p}^{i}+I_{a p p}^{e}\right) /(1+\lambda)$.

The dynamics of $S$ gating variables are described by a so-called membrane model, consisting of ordinary differential equations of the form

$$
\frac{\partial w_{j}}{\partial t}=R_{j}\left(v, w_{j}\right)=\left(w_{j \infty}(v)-w_{j}\right) / \tau_{j}(v), \quad j=1, . ., S .
$$

In this paper, we consider one of the most used detailed membrane models in the literature, the Luo-Rudy phase I (LR1) model (see Luo and Rudy [1991]), based on six gating variables and one variable for the calcium ionic concentration. 


\section{Discretization of the models}

The Monodomain (2) and Bidomain models (1) are discretized by meshing the cardiac tissue volume $H$ with a structured grid of hexahedral isoparametric $Q_{1}$ elements and by introducing the associated finite element space $V_{h}$. A semidiscrete problem is obtained by applying a standard Galerkin procedure and choosing a finite element basis $\left\{\phi_{i}\right\}$ for $V_{h}$. We denote by $\mathrm{M}=\left\{\mathrm{m}_{r s}=\int_{H} \varphi_{r} \varphi_{s} \mathrm{dx}\right\}$ the symmetric mass matrix, by $\mathrm{A}_{m, i, e}=\left\{\mathrm{a}_{r s}^{m, i, e}=\right.$ $\left.\int_{H}\left(\nabla \varphi_{r}\right)^{T} D_{m, i, e} \nabla \varphi_{s} \mathrm{dx}\right\}$ the symmetric stiffness matrices and by $\mathrm{I}_{i o n}^{h}, \mathrm{I}_{a p p}^{(m, i, e), h}$ the finite element interpolants of $I_{i o n}$ and $I_{a p p}^{m, i, e}$, respectively. Integrals are computed with a $3 \mathrm{D}$ trapezoidal quadrature rule, so the mass matrix $M$ is lumped to diagonal form; see Quarteroni and Valli [1994] for an introduction to finite element methods. In our implementation, we have actually reordered the unknowns writing for every node the $\mathbf{u}_{i}$ and $\mathbf{u}_{e}$ components consecutively, so as to minimize bandwidth of the stiffness matrix.

The time discretization is performed by a semi-implicit method using for the diffusion term the implicit Euler method, while the nonlinear reaction term $\mathrm{I}_{\text {ion }}$ is treated explicitly. The use of an implicit treatment of the diffusion terms appearing in the Mono or Bidomain models is essential to allow an adaptive change of the time step according to the stiffness of the various phases of the heartbeat. The ODE system for the gating variables is discretized by the semiimplicit Euler method; in this way we decouple the gating variables by solving the gating system first (given the potential $\mathbf{v}^{n}$ at the previous time-step)

$$
\left(\mathbf{w}^{n+1}-\mathbf{w}^{n}\right) / \Delta t=R\left(\mathbf{v}^{n}, \mathbf{w}^{n+1}\right)
$$

and then solving for $\mathbf{u}_{i}^{n+1}, \mathbf{u}_{e}^{n+1}$ in the Bidomain case

$$
\begin{gathered}
\left(\frac{c_{m}}{\Delta t}\left[\begin{array}{rr}
\mathrm{M} & -\mathrm{M} \\
-\mathrm{M} & \mathrm{M}
\end{array}\right]+\left[\begin{array}{cc}
\mathrm{A}_{i} & 0 \\
0 & \mathrm{~A}_{e}
\end{array}\right]\right)\left(\begin{array}{l}
\mathbf{u}_{i}^{n+1} \\
\mathbf{u}_{e}^{n+1}
\end{array}\right)= \\
\frac{c_{m}}{\Delta t}\left(\begin{array}{l}
\mathrm{M}\left(\mathbf{u}_{i}^{n}-\mathbf{u}_{e}^{n}\right) \\
\mathrm{M}\left[-\mathbf{u}_{i}^{n}+\mathbf{u}_{e}^{n}\right]
\end{array}\right)+\left(\begin{array}{l}
\mathrm{M}\left[-\mathrm{I}_{i o n}^{h}\left(\mathbf{v}^{n}, \mathbf{w}^{n+1}\right)+\mathrm{I}_{a p p}^{i, h}\right] \\
\mathrm{M}\left[\begin{array}{l}
\text { ion } \\
\text { ion }
\end{array}\left(\mathbf{v}^{n}, \mathbf{w}^{n+1}\right)-\mathrm{I}_{a p p}^{e, h}\right]
\end{array}\right),
\end{gathered}
$$

where $\mathbf{v}^{n}=\mathbf{u}_{i}^{n}-\mathbf{u}_{e}^{n}$. As in the continuous model, $\mathbf{v}^{n}$ is uniquely determined, while $\mathbf{u}_{i}^{n}$ and $\mathbf{u}_{e}^{n}$ are determined only up to the same additive time-dependent constant chosen by imposing the condition $\mathbf{1}^{T} \mathrm{Mu}_{e}^{n}=0$.

In the Monodomain case, we have to solve for $\mathbf{v}^{n+1}$

$$
\left(\frac{c_{m}}{\Delta t} \mathrm{M}+\mathrm{A}_{m}\right) \mathbf{v}^{n+1}=\frac{c_{m}}{\Delta t} \mathrm{Mv}^{n}-\mathrm{MI}_{i o n}^{h}\left(\mathbf{v}^{n}, \mathbf{w}^{n+1}\right)+\mathrm{MI}_{a p p}^{m, h} .
$$

We employed an adaptive time-stepping strategy based on controlling the transmembrane potential variation $\Delta v=\max \left(\mathbf{v}^{n+1}-\mathbf{v}^{n}\right)$ at each timestep, see Luo and Rudy [1991]. If $\Delta v<\Delta v_{\text {min }}=0.05$ then we select $\Delta t=\left(\Delta v_{\max } / \Delta v\right) \Delta t$ (if smaller than $\left.\Delta t_{\max }=6 \mathrm{msec}\right)$, if $\Delta v>\Delta v_{\max }=0.5$ then we select $d t=\left(\Delta v_{\min } / \Delta v\right) d t$ (if greater than $\Delta t_{\text {min }}=0.005 \mathrm{msec}$ ). In 
order to guarantee a control on the variation of the gating variables of the LR1 membrane model as well, each gating equation of the system (3) is integrated exactly (see Victorri and et al. [1985]), while the calcium ionic concentration is updated using the explicit Euler method.

The linear system at each time step in the discrete problems is solved iteratively by the preconditioned conjugate gradient (PCG) method, using as initial guess the solution at the previous time step. Parallelization and portability are realized using the PETSc parallel library (Balay et al. [2001]) and a preconditioned conjugate gradient solver at each time step, with block Jacobi preconditioner and ILU(0) on each block, the default one-level preconditioner in the PETSc library. The numerical experiments reported in the next section show that it performs well in the Monodomain case, but not in the Bidomain case. Therefore, more research is needed in order to build better preconditioners, particularly with two or more levels; see Smith et al. [1996].

\section{Numerical results}

We have conducted several numerical experiments in three dimensions on distributed memory parallel architectures, with both the Monodomain and the Bidomain model coupled with the LR1 membrane model. The parallel machines employed are an IBM SP RS/6000 with 512 processors Power 4 of the Cineca Consortium (www.cineca.it), and an HP SuperDome 64000 with 64 processors PA8700 of the Cilea Consortium (www.cilea.it). We refer to Colli Franzone and Pavarino [2003] for more detailed numerical results. Multigrid preconditioners for the Bidomain system have been studied by Weber dos Santos et al. [2004], while mortar finite element discretizations by Pennacchio [2004]. We studied first the spectrum of the iteration matrices (5) and (4) on a small $15 \times 15 \times 8$ mesh in the Monodomain case and $15 \times 15 \times 4$ in the Bidomain case (these meshes are chosen in order to have matrices of the same size). The eigenvalues of the stiffness matrices are reported in the left panel of Figure 1, while the eigenvalues of the iteration matrices are reported in the right panel. It is clear that the addition to the stiffness matrix of a term with the mass matrix greatly improves the spectrum of the Monodomain iteration

Table 1. Parameters calibration for numerical tests

\begin{tabular}{|ll|}
\hline ellipsoidal & $a_{1}=b_{1}=1.5 \mathrm{~cm}, a_{2}=b_{2}=2.7 \mathrm{~cm}, c_{1}=4.4, c_{2}=5 \mathrm{~cm}$ \\
geometry & $\phi_{\min }=0, \phi_{\max }=2 \pi, \theta_{\min }=-3 \pi / 8, \theta_{\max }=\pi / 8$ \\
\hline \multicolumn{3}{|c|}{$\chi=10^{3} \mathrm{~cm}^{-1}, C_{m}=10^{-3} \mathrm{mF} / \mathrm{cm}^{2}$} \\
\hline Monodomain $\sigma_{l}=1.2 \cdot 10^{-3} \Omega^{-1} \mathrm{~cm}^{-1}, \sigma_{t}=2.5562 \cdot 10^{-4} \Omega^{-1} \mathrm{~cm}^{-1}$ \\
parameters & $G=1.5 \Omega^{-1} \mathrm{~cm}^{-2}, v_{t h}=13 \mathrm{mV}, v_{p}=100 \mathrm{mV}$ \\
& $\eta_{1}=4.4 \Omega^{-1} \mathrm{~cm}^{-2}, \quad \eta_{2}=0.012, \quad \eta_{3}=1$ \\
\hline \multirow{3}{*}{ Bidomain } & $\sigma_{l}^{e}=2 \cdot 10^{-3} \Omega^{-1} \mathrm{~cm}^{-1}, \sigma_{l}^{i}=3 \cdot 10^{-3} \Omega^{-1} \mathrm{~cm}^{-1}$ \\
parameters & $\sigma_{t}^{e}=1.3514 \cdot 10^{-3} \Omega^{-1} \mathrm{~cm}^{-1}, \sigma_{t}^{i}=3.1525 \cdot 10^{-4} \Omega^{-1} \mathrm{~cm}^{-1}$ \\
& $\sigma_{n}^{e}=\sigma_{t}^{e} / \mu_{1}, \sigma_{n}^{i}=\sigma_{t}^{i} / \mu_{2}$ \\
& $\mu_{1}=\mu_{2}=1$ axial isotropic case, $\mu_{1}=2, \mu_{2}=10$ orthotropic case \\
\hline
\end{tabular}



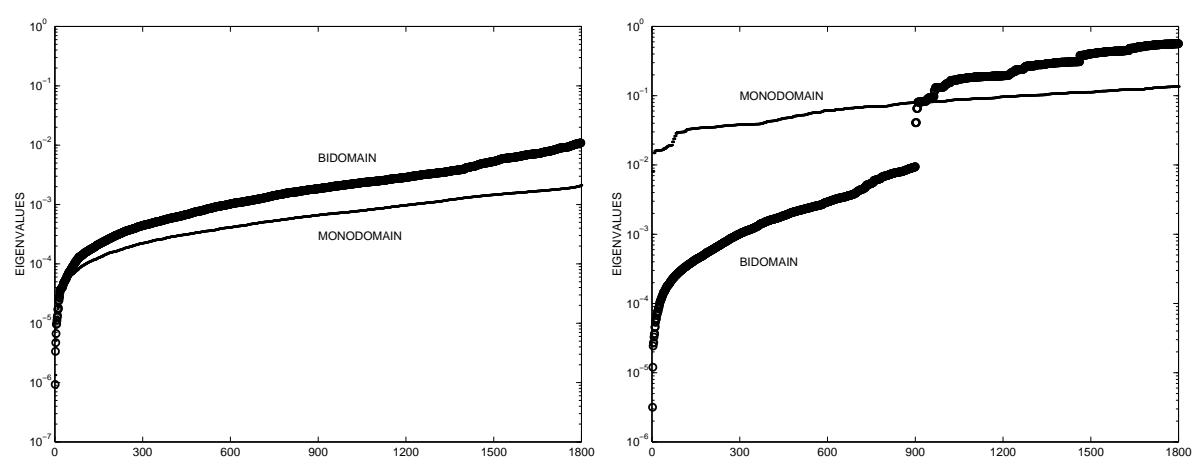

Fig. 1. Nonzero eigenvalue distribution of the stiffness matrices related to elliptic operators with Neumann boundary conditions (left) and of the iteration matrices in (5) and (4) (right) for a small mesh. Monodomain eigenvalues are denoted by dots $(\cdot)$, Bidomain eigenvalues by circles (o)

matrix (5), but not of the Bidomain iteration matrix (4). In fact, the iterative solution of the linear system at each time step turns out to be much harder for the Bidomain model than for the Monodomain model.

\subsection{Scaled speedup for Monodomain-LR1 and Bidomain-LR1 solvers}

We consider first the Monodomain equation with LR1 ionic model, simulating on the IBM SP4 machine the initial depolarization of some ellipsoidal blocks after one stimulus of $250 \mathrm{~mA} / \mathrm{cm}^{3}$ has been applied for 1 msec on a small area (5 mesh points in each direction) of the epicardium. The blocks are chosen in increasing sizes so as to keep constant the number of mesh points per subdomain (processor). As shown in Figure 2, the domain varies from the smaller block with 8 subdomains to half ventricle with 128 subdomains. We fixed the local mesh in each subdomain to be of $75 \times 75 \times 50$ nodes $(281.750$ unknowns), hence varying the global number of unknowns of the linear system from $2.25 \cdot 10^{6}$ in the smaller case with 8 subdomains on a global mesh of $150 \times 150 \times 100$ nodes to $3.6 \cdot 10^{7}$ in the larger case with 128 subdomains on a global mesh of $600 \times 600 \times 100$ nodes. The model is run for 30 time steps of $0.05 \mathrm{msec}$ each. At each time step, we compute the potential $v$, the gating and concentration variables $w_{1}, \cdots, w_{7}$ and the depolarization time. The results are reported in the upper part of Table 2. The assembling time, average number of PCG iterations per time step and the average time per time step (last three columns) are reasonably small. Up to 64 processors, the algorithm seems practically scalable, and even for 128 processors, the number of PCG iterations grows to just 8 .

We then consider the Bidomain system with LR1 ionic model, in the same setting (initial stimulus and domain decomposition) of the previous case. At 

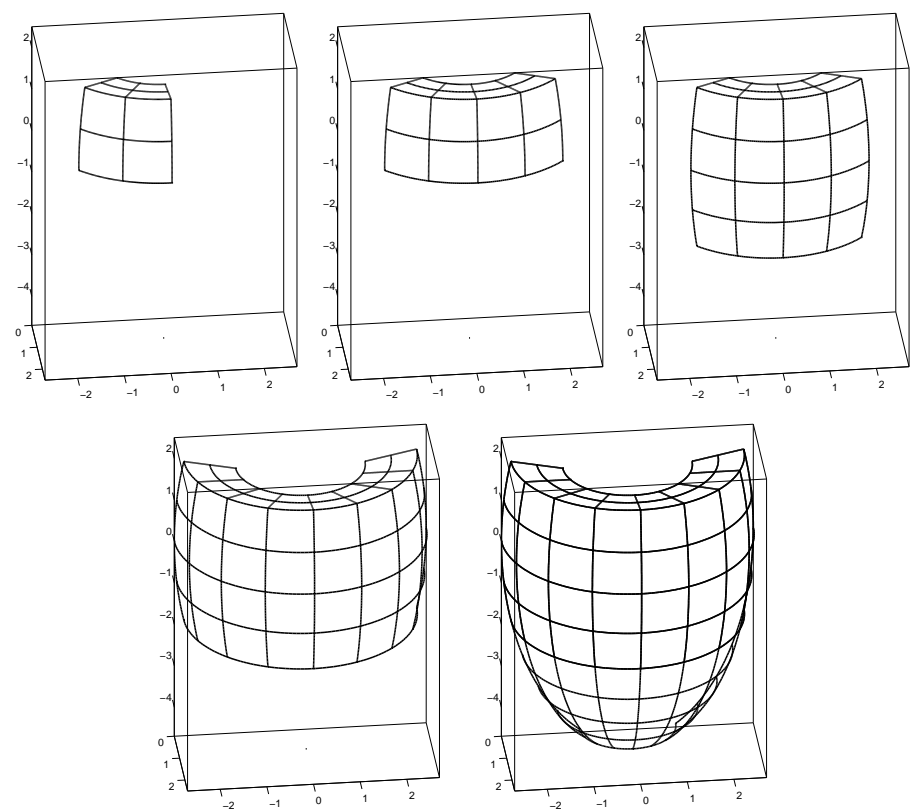

Fig. 2. Scaled speedup test: ellipsoidal domains of increasing sizes decomposed into $8,16,32,64$ and 128 subdomains of fixed size

Table 2. Scaled speedup tests for Monodomain - LR1 and Bidomain - LR1 models. Initial depolarization of an ellipsoidal block: 1 stimulus on epicardial surface, 30 time steps of 0.05 msec each, computation of $v, w_{1}, \cdots, w_{7}$ and isochrones. $t_{A}=$ assembly timing, it $=$ average number of PCG iterations at each time step, time $=$ average CPU timing of each time step

\begin{tabular}{|r|c|r|r|r|r|}
\hline \multicolumn{7}{|c|}{ Monodomain - LR1 } \\
\hline \# proc. & mesh & $\begin{array}{c}\text { unknowns } \\
\text { (nodes) }\end{array}$ & $t_{A}$ & it. & time \\
\hline $8=2 \cdot 2 \cdot 2$ & $150 \times 150 \times 100$ & 2.250 .000 & $7.7 \mathrm{~s}$ & 4 & $2.7 \mathrm{~s}$ \\
$16=4 \cdot 2 \cdot 2$ & $300 \times 150 \times 100$ & 4.500 .000 & $8.5 \mathrm{~s}$ & 4 & $3 \mathrm{~s}$ \\
$32=4 \cdot 4 \cdot 2$ & $300 \times 300 \times 100$ & 9.000 .000 & $9.1 \mathrm{~s}$ & 5 & $3.6 \mathrm{~s}$ \\
$64=8 \cdot 4 \cdot 2$ & $600 \times 300 \times 100$ & 18.000 .000 & $9.2 \mathrm{~s}$ & 5 & $3.6 \mathrm{~s}$ \\
$128=8 \cdot 8 \cdot 2$ & $600 \times 600 \times 100$ & 36.000 .000 & $10.6 \mathrm{~s}$ & 8 & $5.1 \mathrm{~s}$ \\
\hline \hline \multicolumn{7}{|c|}{ Bidomain - LR1 } \\
\hline \# proc. & mesh & unknowns & $t_{A}$ & it. & time \\
\hline $8=2 \cdot 2 \cdot 2$ & $100 \times 100 \times 70$ & 1.400 .000 & $12.9 \mathrm{~s}$ & 98 & $40.2 \mathrm{~s}$ \\
$16=4 \cdot 2 \cdot 2$ & $200 \times 100 \times 70$ & 2.800 .000 & $13.3 \mathrm{~s}$ & 127 & $55.5 \mathrm{~s}$ \\
$32=4 \cdot 4 \cdot 2$ & $200 \times 200 \times 70$ & 5.600 .600 & $15.7 \mathrm{~s}$ & 148 & 72 \\
$64=8 \cdot 4 \cdot 2$ & $400 \times 200 \times 70$ & 11.200 .000 & $16.2 \mathrm{~s}$ & 176 & $91.9 \mathrm{~s}$ \\
$128=8 \cdot 8 \cdot 2$ & $400 \times 400 \times 70$ & 22.400 .000 & $18.4 \mathrm{~s}$ & 244 & $129.7 \mathrm{~s}$ \\
\hline
\end{tabular}


each time step, we now compute the potentials $u_{i}, u_{e}$, the gating and concentration variables and the depolarization time. Due to the larger memory requirements of the Bidomain model, we used a smaller mesh of $50 \times 50 \times 35$ nodes in each subdomain (processor), hence varying the global number of unknowns of the linear system from $1.4 \cdot 10^{6}$ in the smaller case with 8 subdomains on a global mesh of $100 \times 100 \times 70$ to $2.24 \cdot 10^{7}$ unknowns in the larger case with 128 subdomains on a global mesh of $400 \times 400 \times 70$ nodes. The results are reported in the lower part of Table 2. While the assembling time remains reasonable (under 20 sec.), the average number of PCG iterations per time step and the average time per time step are now much larger, clearly showing the limits of the one-level preconditioner and the effects of the severe ill-conditioning of the Bidomain iteration matrix.

\subsection{Simulation of a full cardiac cycle}

We also simulated a complete cardiac cycle (excitation-recovery) in a slab of cardiac tissue of size $2 \times 2 \times 0.5 \mathrm{~cm}^{3}$, discretized with a fine mesh $200 \times 200 \times 50$. We used 25 processors of the HP SuperDome machine with 64 processors. The fibers rotate intramurally linearly with depth for a total amount of $90^{\circ}$. A stimulus is applied at an epicardial vertex and the excitation of the entire slab requires about $80 \mathrm{msec}$, while the time interval for simulating the cardiac cycle is on the order of $360 \mathrm{msec}$. The adaptive time-stepping algorithm automatically adapts, in an efficient way, the time step size in the three main different phases of the heart beat, see Figure 3 (left). While the Monodomain solver is quite efficient, the Bidomain solver is not, since the number of PCG iterations at each time step increases considerably, reaching a maximum of about 250 iterations in the depolarization phase, see Figure 3 (right). The simulation took about 6.4 days for the the Bidomain model and about 5 hours for the Monodomain model. We compared the two computer platforms mentioned above by simulating the Monodomain model on a slab
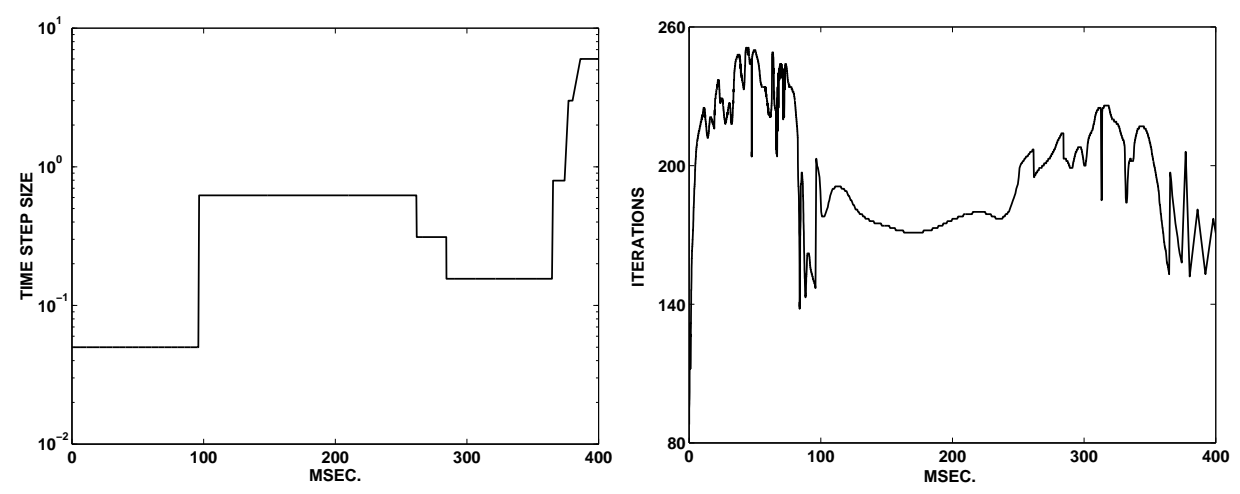

Fig. 3. Full cardiac cycle with Bidomain model and LR1 gating. Time-step size in msec. on a semilogarithmic scale (left), PCG iterations at each time step (right) 
with dimensions $4 \times 4 \times 0.5 \mathrm{~cm}^{3}$ and mesh $400 \times 400 \times 50$ : the HP SuperDome machine with 32 processors took about 20 hours and the IBM SP4 machine with 64 processors took about 2.5 hours. Therefore, a considerable CPU time reduction in the Bidomain case is to be expected by using the SP4 machine.

\section{References}

S. Balay, W. D. Gropp, L. C. McInnes, and B. F. Smith. PETSc users manual. Technical Report ANL-95/11 - Revision 2.1.1, Argonne National Laboratory, 2001.

P. Colli Franzone and L. F. Pavarino. A parallel solver for reaction-diffusion systems in computational electrocardiology. Technical report, IMATI CNR Tech. Rep. 9-PV, 2003.

P. Colli Franzone and G. Savaré. Degenerate evolution systems modeling the cardiac electric field at micro and macroscopic level. In A. Lorenzi and B. Ruf, editors, Evolution equations, Semigroups and Functional Analysis, pages 49-78. Birkhauser, 2002.

J. Keener and J. Sneyd. Mathematical Physiology. Springer, 1998.

I. LeGrice and et al. Laminar structure of the heart: ventricular myocyte arrangement and connective tissue architecture in the dog. Am. J. Physiol. (Heart Circ. Physiol), 269 (38):H571-H582, 1995.

C. Luo and Y. Rudy. A model of the ventricular cardiac action potential: depolarization, repolarization, and their interaction. Circ. Res., 68 (6): 1501-1526, 1991.

M. Pennacchio. The mortar finite element method for the cardiac bidomain model of extracellular potential. J. Sci. Comp., 20 (2), 2004. To appear.

A. Quarteroni and A. Valli. Numerical Approximation of Partial Differential Equations. Springer-Verlag, Berlin, 1994.

B. F. Smith, P. E. Bjørstad, and W. Gropp. Domain Decomposition: Parallel Multilevel Methods for Elliptic Partial Differential Equations. Cambridge University Press, 1996.

D. Streeter. Gross morphology and fiber geometry in the heart. In R. Berne, editor, Handbook of Physiology, vol. 1 The Heart, pages 61-112. Williams \& Wilkins, Baltimore, 1979.

A. Victorri and et al. Numerical integration in the reconstruction of cardiac action potential using the Hodgkin-Huxley type models. Comp. Biomed. Res., 18:10-23, 1985.

R. Weber dos Santos, G. Plank, B. S., and V. E.J. Preconditioning techniques for the bidomain equations. In R. K. et al., editor, These Proceedings. LNCSE. Springer, 2004. 